\title{
Intensity of arterial structure acquired by Silent MRA estimates cerebral blood flow
}

\author{
Zhen-An Hwang ${ }^{1,2}$, Chia-Wei Li ${ }^{1,3}$, Chien-Yuan Eddy Lin ${ }^{3}$, Jyh-Horng Chen ${ }^{4,5}$, Chia-Yuen Chen ${ }^{1,2}$ and
}

Wing P. Chan ${ }^{1,2^{*}}$ (D)

\begin{abstract}
Background: Cerebral blood flow (CBF) and the morphology of the cerebral arteries are important for characterizing cerebrovascular disease. Silent magnetic resonance angiography (Silent MRA) is a MRA technique focusing on arterial structural delineation. This study was conducted to investigate the correlation between Silent MRA and CBF quantification, which has not yet been reported.
\end{abstract}

Methods: Both the Silent MRA and time-of-flight magnetic resonance angiography scans were applied in seventeen healthy participants to acquire the arterial structure and to find arterial intensities. Phase-contrast MRA (PC-MRA) was then used to perform the quantitative CBF measurement of 13 cerebral arteries. Due to different dataset baseline signal level of Silent MRA, the signal intensities of the selected 13 cerebral arteries were normalized to the selected ROls of bilateral internal carotid arteries. The normalized signal intensities were used to determine the relationship between Silent MRA and CBF.

Results: The image intensity distribution of arterial regions generated by Silent MRA showed similar laminar shape as the phase distribution by PC-MRA (correlation coefficient $>0.62$ ). Moreover, in both the results of individual and group-leveled analysis, the intensity value of arterial regions by Silent MRA showed positively correlation with the CBF by PC-MRA. The coefficient of determination $\left(R^{2}\right)$ of individual trends ranged from 0.242 to 0.956 , and the $R^{2}$ of groupleveled result was 0.550 .

Conclusions: This study demonstrates that Silent MRA provides valuable CBF information despite arterial structure, rendering it a potential tool for screening for cerebrovascular disease.

Keywords: Blood flow, Brain, Magnetic resonance angiography (MRA), Magnetic resonance imaging (MRI)

\section{Keypoints}

- Imaging assessment of intracranial arteries and cerebral blood flow is essential in cerebrovascular diagnosis.

- Conventional TOF-MRA for evaluating intracranial arteries still faces some limitations and artifacts.

\footnotetext{
*Correspondence: wingchan@tmu.edu.tw

1 Department of Radiology, Wan Fang Hospital, Taipei Medical University,

111 Hsing Long Road, Section 3, Taipei 116, Taiwan

Full list of author information is available at the end of the article
}

- Compared with TOF-MRA, Silent MRA provides additional cerebral blood flow information despite arterial structure

\section{Background}

Evaluating cerebral hemodynamics, including vessel morphology and flow information, is important for characterizing the pathological features of acute stroke, chronic cerebrovascular disease, epilepsy, and brain tumors. In particular, morphological assessments of intracranial arteries are essential in cerebrovascular disease diagnosis. Therefore, imaging techniques focusing 
on intracranial artery delineation and cerebral blood flow (CBF) have been developed and applied clinically [1].

Conventionally, three-dimensional time-of-flight magnetic resonance angiography (3D TOF-MRA) has been used for evaluating morphology of intracranial arteries [2]. This contrast-agent-free technique uses the flow of spins to generate vessel contrast; it is sometimes considered as a follow-up imaging alternative to digital subtraction angiography [3]. Unfortunately, spin saturation effects (in slow flow) and phase dispersion artifacts (in turbulent flow) decrease signal intensity in 3D TOFMRA [4-6], leading to overestimation of the severity of intracranial artery disease. Additionally, 3D TOF-MRA is prone to magnetic susceptibility and radiofrequency shielding artifacts. In addition, cerebral flow rate cannot be determined using 3D TOF-MRA.

As a result, phase-contrast quantitative magnetic resonance angiography (PC-MRA) without contrast agent has been developed to measure $\mathrm{CBF}$ as a volume flow rate $(\mathrm{ml} / \mathrm{min})[7,8]$. Zarrinkoob et al. [9] reported the distribution of total cerebral arterial flow across variations in age, sex, and anatomy using high-resolution PC-MRA establishing a normative reference value for blood flow in major cerebral arteries in healthy people [7]. Although PC-MRA can provide multidirectional flow and collateral flow, it does not improve stenosis detection in major intracranial vessels compared to 3D TOF-MRA because the optimized velocity-encoding gradient affects predominantly small vessels and the reduced number of partitions [10].

Silent magnetic resonance angiography (Silent MRA) is another MRA imaging technique [11-13]. As with 3D TOF-MRA, Silent MRA does not require contrast agent; it is a non-invasive perfusion imaging technique that uses continuous arterial spin labeling with a long radiofrequency inversion pulse, and it labels blood within the carotid arteries as an endogenous tracer [14, 15]. Subtracted by another image dataset without the use of arterial spin labeling, Silent MRA can depict the intracranial arteries without background tissues. However, with arterial spin labeling technique, Silent MRA tends to have drop-off on inflow enhancement [16]. Previous study reported that Silent MRA showed low signal intensity in distal vessels because of poor inflow (two of 27 intracranial Silent Scans) [17]. On the other hand, it implied that the signal intensity obtained by Silent MRA contained not only the structure of arteries but also the flow information.

Moreover, with the use of a zero echo time technique, Silent MRA is able to minimize the phase dispersion of the labeled blood flow signal and decrease magnetic susceptibility compared to 3D TOF-MRA [18]. Its use has recently been proposed for assessing vascular lesions such as those in treated intracranial aneurysms [18-21] and in cerebral arteriovenous malformations [22, 23]. These studies show that it provides excellent architectural visualization in coiled aneurysms and flow through intracranial stents.

Studies have focused primarily on delineating vascular lesion structures using a combined zero-echo-time and arterial spin labeling technique, but none have thoroughly investigated the use of Silent MRA for estimating CBF. Therefore, we aimed to explore the usefulness of Silent MRA for flow estimation. We studied the correlation between Silent MRA and CBF as estimated using PC-MRA in a healthy population.

\section{Methods \\ Participants}

Seventeen healthy volunteers (8 women; mean age, $33.8 \pm 7.1$ years) joined this study between October 2018 and July 2019. None had claustrophobia, psychological disorders, cardiac pacemakers, contraindications to magnetic resonance imaging, or metal implants, and none were pregnant. During the entire scanning session, the participants were to maintain a motionless head. Participants remained awake to prevent unwanted motion artifacts. This study was approved by the Research Ethics Committee of Taipei Medical University-Joint Institutional Review Board (N201803017), and informed consent was obtained from all participants.

\section{MRA scanning}

All magnetic resonance images were acquired using a 3.0-T clinical scanner (Discovery MR750w; GE Healthcare, Milwaukee, USA) equipped with a 24-channel Geometry Embracing Method head-and-neck coil for signal detection and a whole-body coil for radio-frequency excitation. Both the Silent MRA and 3D TOF-MRA scans were used to acquire the arterial structure before performing 2D PC-MRA. The parameters for acquiring the Silent MRA were: repetition time, $759.924 \mathrm{~ms}$; echo time, $0.04 \mathrm{~ms}$; flip angle, $5^{\circ}$; field of view, $20 \mathrm{~cm}$; matrix, $256 \times 256$; section thickness, $1.4 \mathrm{~mm}$; NEX, 1.0; bandwidth, $62.5 \mathrm{kHz}$; and acquisition time, $5 \min 15 \mathrm{~s}$. The parameters for acquiring the 3D TOF-MRA were: repetition time/echo time, $19 \mathrm{~ms} / 2.9 \mathrm{~ms}$; flip angle, $15^{\circ}$; field of view, $18 \mathrm{~cm}$; matrix, $416 \times 192$; section thickness, $1.2 \mathrm{~mm}$; NEX, 1; bandwidth, $41.7 \mathrm{kHz}$; and acquisition time, $3 \mathrm{~min} 31 \mathrm{~s}$.

In the 2D PC-MRA flow-measuring scan, a NonInvasive Optimal Vessel system (NOVA; VasSol, Inc., Chicago, IL, USA) was used to perform the quantitative flow measurements in 13 cerebral arteries: the basilar artery, the bilateral anterior cerebral arteries, the superior branches of the bilateral anterior cerebral arteries, 
the bilateral middle cerebral arteries, the bilateral posterior cerebral arteries, the bilateral vertebral arteries, and the bilateral internal carotid arteries. The parameters for acquiring the PC MRA were: repetition time/echo time, $12.82 \mathrm{~ms} / 5.728 \mathrm{~ms}$; flip angle, $25^{\circ}$; field of view, $16 \mathrm{~cm}$; matrix, $256 \times 256$; section thickness, $5 \mathrm{~mm}$; NEX, 1 ; bandwidth, about $162.734 \mathrm{kHz}$; VEC, automatically selected by NOVA software. To minimize the effects of turbulence, flow was measured at the point furthest from vessel turns within the designated segment. Perpendicular imaging for any given vessel was automatically created by the NOVA system so that arterial flow could be accurately measured. A laminar flow model was used to calculate vascular flow rates. The vessels used to designate flow status were those in distal territories of the vertebrobasilar tree. Quantitative flow was then measured in the chosen vessels via the 2D PC-MRA technique using the NOVA system. Data were acquired under the guidance of one experienced (25 years) radiological technologist.

\section{Silent MRA data analysis}

We compared the arterial structure and vessel uniformity of bilateral internal carotid arteries (ICAs) between Silent MRA and 3D TOF-MRA. To interpret the flow information obtained using Silent MRA, average image intensities based on the exact location of the 13 cerebral arteries in PC imaging were calculated, and the flow-encoding Silent MRA signal distribution was compared with that obtained using PC flow imaging. Because the baseline of each individual Silent MRA dataset resulted in a different signal level, the image intensities of the 13 arterial areas were normalized to the central regions of the selected ROIs located prior to the bilateral cervical segments of ICAs (Fig. 1). In individual analyses, the normalized intensities of the arterial regions, generated using Silent MRA, were correlated to the corresponding flow rates generated using PC imaging. In the group-level analysis, one linear regression was applied across all the normalized arterial intensities and their corresponding flow rates, allowing investigation of the relationship between the normalized arterial intensities and CBF. To explore the relationship between the normalized arterial intensity from the Silent MRA and the quantified CBF, linear regression with $95 \%$ confidence intervals was calculated. Statistical analyses were performed using $\mathrm{R}$ version 3.5.1 (R Core Team 2018. R: A language and environment for statistical computing. R Foundation for Statistical Computing, Vienna, Austria. URL http://www.R-project.org/).

\section{Results}

Compared with 3D TOF-MRA, Silent MRA provided more detail in the arterial structure and a more uniform intensity in the petrous segments of internal carotid arteries (one example was listed in Fig. 2, and all the dataset were listed in the Additional file 1: Fig. S1), and it showed comparatively less signal loss in the Silent MRA. Additionally, its structural map showed gradient

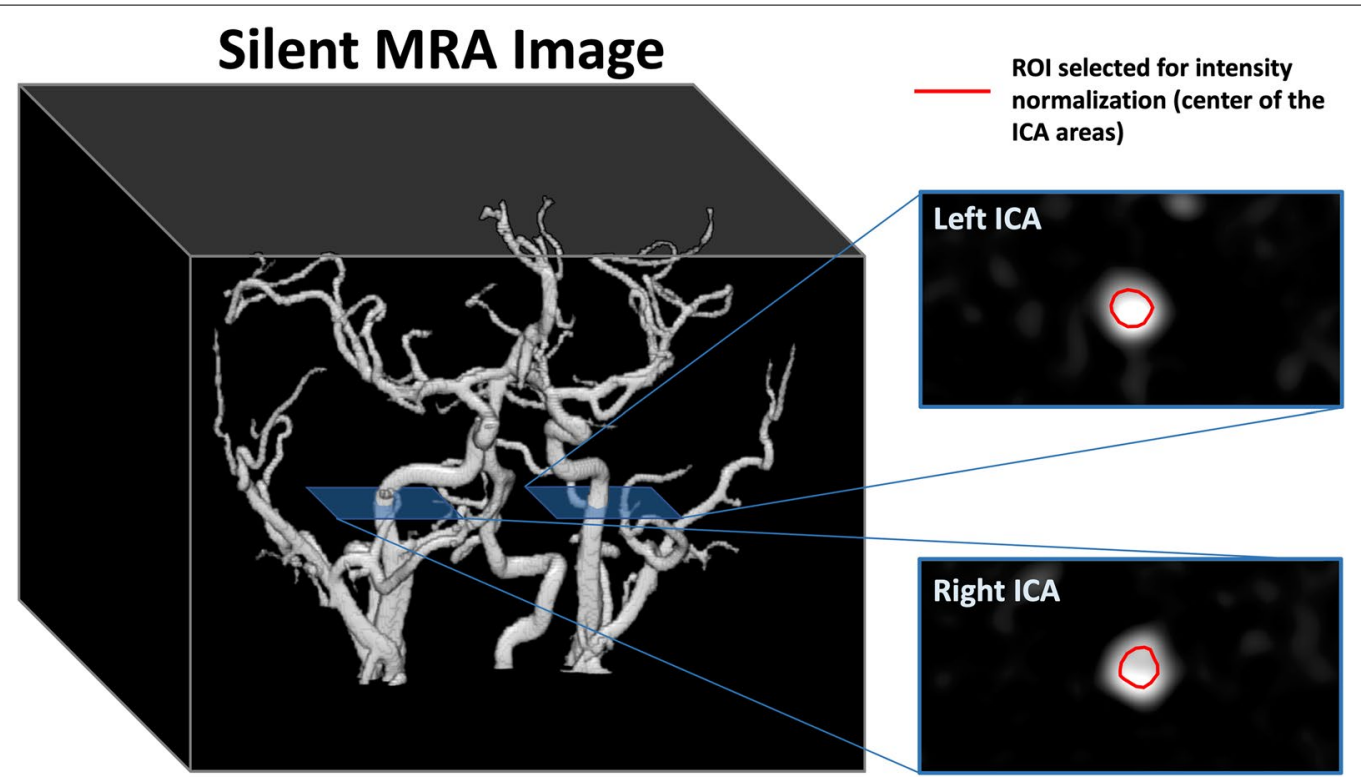

Fig. 1 Locations of selected ROls (marked with red lines) based on bilateral cervical segments of internal carotid arteries (ICA) for intensity normalization. The magnitudes of the individual Silent MRA data were divided by the average intensity value of the two selected ROls for the following group-level analysis 


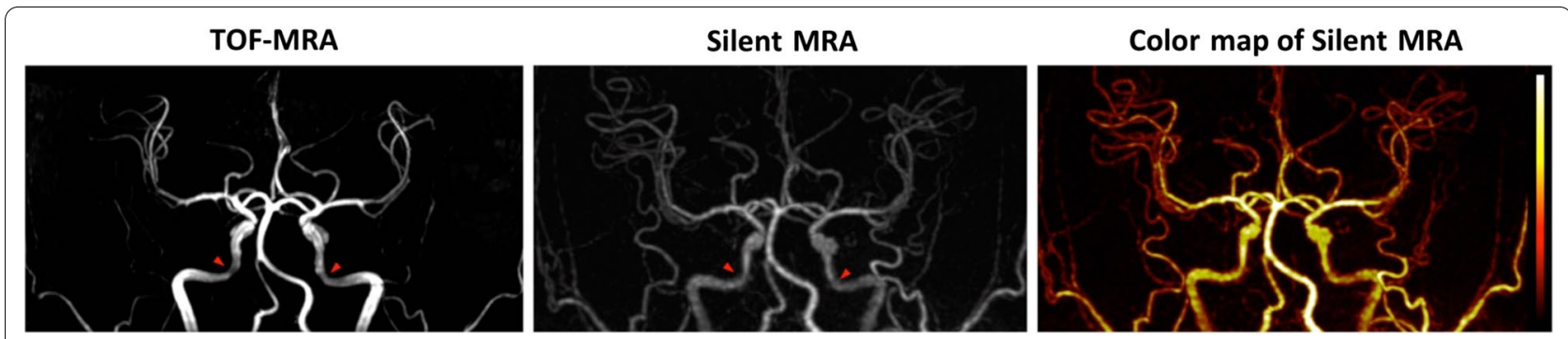

Fig. 2 Arterial structures acquired using Silent MRA and 3D TOF-MRA. Red arrows indicate the petrous segments of internal carotid arteries. Silent MRA shows comparatively less signal loss. Using the arterial spin-labeling technique, Silent MRA provides flow information in the gradient magnitudes of the arterial regions

intensities that resulted from flow-weighted information, such as laminar-shaped flows, in the arterial regions. Figure 3 shows the demographic data of the laminar-shaped phase distribution via 2D PC-MRA and the intensity variations of the 13 cerebral arteries via Silent MRA. All phase distributions acquired using 2D PC-MRA yielded a laminar shape, and the intensity distribution shown by Silent MRA agreed with that shape (correlation coefficients of arterial flow-shapes by 2D PC-MRA and Silent MRA are all larger than 0.6). The intensity variation shown by Silent MRA highly correlated with the distribution of phase shown by 2D PC-MRA; their correlation coefficients ranged from 0.62 to 0.98 .

Table 1 compares the CBF values generated using 2D PC-MRA for each of the 13 arteries to the image intensities generated using Silent MRA at the same arterial location. The normalized arterial intensity was adopted at each arterial location in the group-level analysis, and a linear relationship $\left(R^{2}=0.550\right)$ was found (Fig. 4). For every participant, the normalized arterial intensities across the 13 arterial regions were highly positively correlated with the corresponding CBFs: $R^{2}$ ranged from 0.242 to 0.956 . Linear regression was also used to determine the relationship between the normalized arterial intensities in the Silent MRAs and the quantified CBFs. With 95\% confidence, the group-level result predicts the $\mathrm{CBF}$ range when using the following:

$\mathrm{CBF}$ range $($ within $95 \%$ confidence interval $)=\left\{\begin{array}{l}(\text { upper value }) 292.1-7.479 \times \mathrm{SNR}_{\text {silent-MRA }} \\ \text { (lower value } 228.9-51.67 \times \mathrm{SNR}_{\text {silent-MRA }}\end{array}\right.$
Expectedly, Silent MRA provides more details in the arterial structure and a more uniform intensity in the petrous segments of the internal carotid arteries compared to 3D TOF-MRA. Fujiwara et al. [24] similarly showed it was superior to 3D TOF-MRA in carotid artery uniformity and blood vessel contrast. Our results show that with the advantages of arterial spin labeling and zero echo time, Silent MRA provides image intensity distributions that are highly correlated with the phase distributions generated by 2D PC-MRA in these arterial sections. The two demonstrate similar laminar shapes in the studied vessel sections. Moreover, the normalized arterial intensities in Silent MRA are highly positively correlated with the flow rates provided by 2D PC-MRA at the individual participant level; at the group level, results are highly linear as well. Using linear regression and the 95\% confidence interval, the normalized arterial intensity provided by Silent MRA can be indirectly used to predict the corresponding CBF range in these arterial regions. For example, a normalized arterial intensity of 0.7 in the Silent MRA image corresponds, with $95 \%$ confidence, to a CBF ranging from 192.1 to $286.9 \mathrm{ml} / \mathrm{min}$. Therefore, one 5-min Silent MRA can provide both the structural information and a relative quantification of CBF from a 2D PC-MRA, implying its potential role in screening for arterial disease.

\section{Discussion}

The novel contributions of this study are its investigation into the flow-weighted image intensity of Silent MRA, and the determination of a relatively straightforward linear relationship that flexibly connects the normalized arterial intensities in Silent MRA with quantified CBF.
Studies examining the relationship between Silent MRA and 2D PC-MRA have not yet been discussed in the literature, and our study shows that Silent MRA can provide more flow information than 3D TOF-MRA. Conventionally, a low intraluminal signal intensity on a 3D TOF-MRA could be the result of a slow flow state such as a stenosis or occlusion, but this modality reportedly overestimates the degree of intracranial artery stenosis [25]. Additionally, 


\section{Flow shape by Silent MRA and PC-MRA}

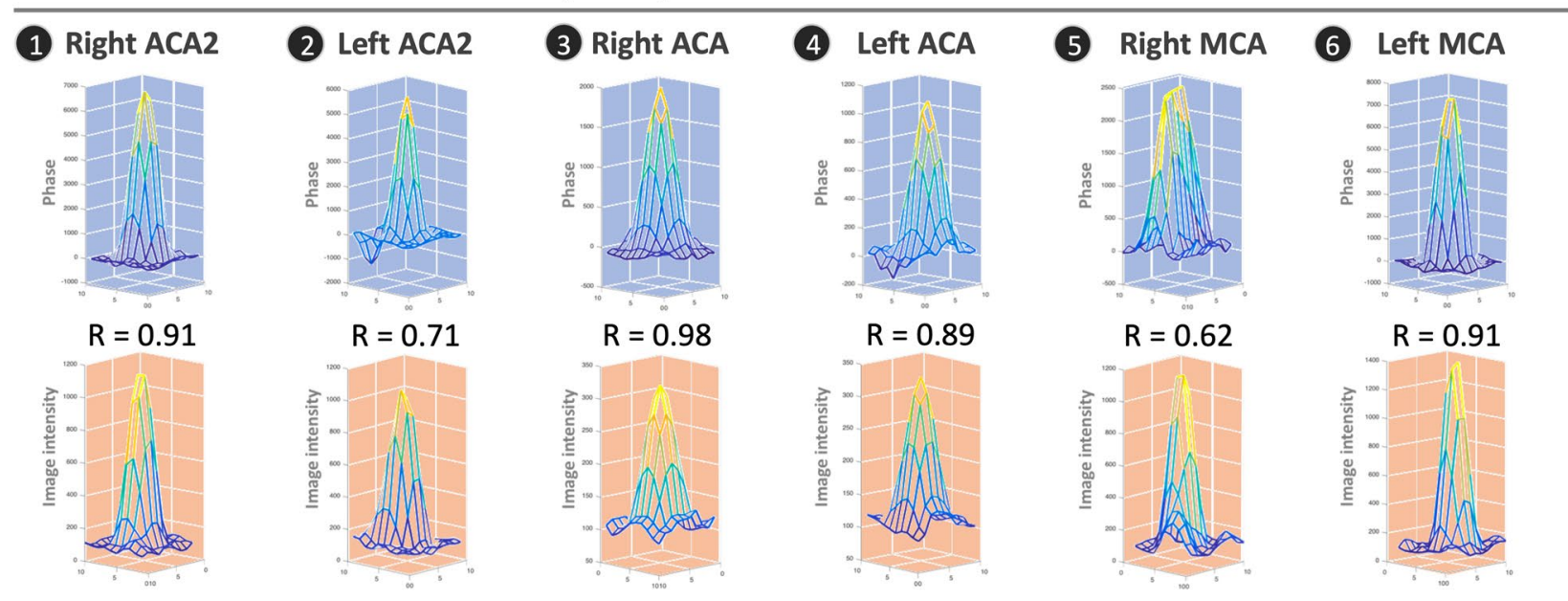

7 Right PCA
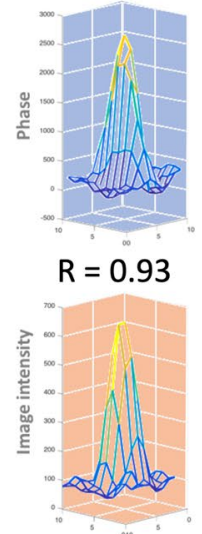

8 Left PCA
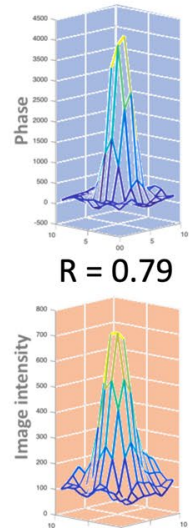

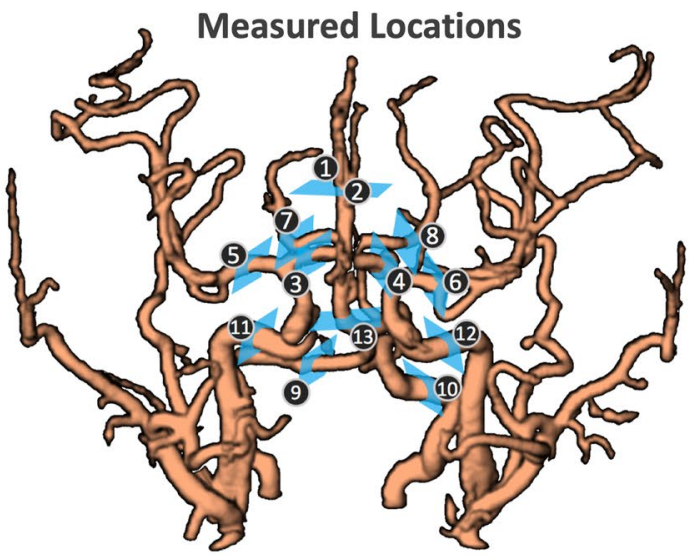

(10) Left VA

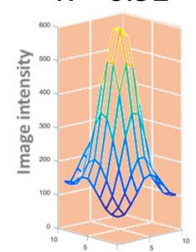

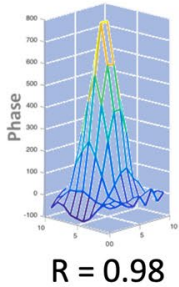

(11) Right ICA



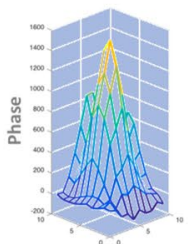

$\mathrm{R}=0.82$

(12) Left ICA


Phase by

PC-MRA

Intensity by

Silent MRA

Fig. 3 Comparisons of flow shapes generated by Silent MRA and 2D PC-MRA. The laminar flow shapes of the 13 cerebral arteries of one study participant (33-years-old; man) are similar whether generated by 2D PC-MRA or Silent MRA. The variable R is the correlation coefficient between the phase distribution (in 2D PC-MRA) and signal intensity (in Silent MRA) 
Table 1 Image intensities in silent MRA and corresponding flow rates determined using 2D PC-MRA

\begin{tabular}{|c|c|c|c|c|c|c|c|c|c|c|c|c|c|c|}
\hline Participant & & $\begin{array}{l}R \\
A \\
C \\
A \\
2\end{array}$ & $\begin{array}{l}\mathrm{L} \\
A \\
C \\
A \\
2\end{array}$ & $\begin{array}{l}R \\
A \\
C \\
A\end{array}$ & $\begin{array}{l}\mathrm{L} \\
\mathrm{A} \\
\mathrm{C} \\
\mathrm{A}\end{array}$ & $\begin{array}{l}R \\
M \\
C \\
A\end{array}$ & $\begin{array}{l}\mathrm{L} \\
M \\
\mathrm{C} \\
\mathrm{A}\end{array}$ & $\begin{array}{l}R \\
P \\
C \\
A\end{array}$ & $\begin{array}{l}L \\
P \\
C \\
A\end{array}$ & $\begin{array}{l}R \\
V \\
A\end{array}$ & $\begin{array}{l}L \\
V \\
A\end{array}$ & $\begin{array}{l}\text { R } \\
\text { I } \\
\text { C } \\
\text { A }\end{array}$ & $\begin{array}{l}\mathrm{L} \\
\mathrm{I} \\
\mathrm{C} \\
\mathrm{A}\end{array}$ & $\begin{array}{l}\text { B } \\
\text { A }\end{array}$ \\
\hline \multirow[t]{2}{*}{1} & Silent MRA & 759 & 755 & 832 & 964 & 1231 & 1308 & 622 & 665 & - & 1164 & 1509 & 1342 & 1200 \\
\hline & Flow rate & 95 & 92 & 127 & 152 & 213 & 241 & 116 & 110 & - & 188 & 417 & 280 & 197 \\
\hline \multirow[t]{2}{*}{2} & Silent MRA & 566 & 585 & 599 & 659 & 1001 & 1157 & 533 & 560 & 521 & 1066 & 1168 & 1040 & 918 \\
\hline & Flow rate & 76 & 83 & 114 & 124 & 158 & 223 & 90 & 78 & 33 & 173 & 328 & 188 & 107 \\
\hline \multirow[t]{2}{*}{3} & Silent MRA & 444 & 704 & 581 & 791 & 981 & 1020 & 410 & 455 & 642 & 697 & 1283 & 1285 & 1074 \\
\hline & Flow rate & 66 & 87 & 93 & 138 & 153 & 150 & 100 & 98 & 73 & 115 & 229 & 242 & 157 \\
\hline \multirow[t]{2}{*}{4} & Silent MRA & 605 & 538 & 697 & 638 & 1122 & 1136 & 341 & 689 & 1023 & 1085 & 1281 & 1373 & 1113 \\
\hline & Flow rate & 70 & 55 & 63 & 50 & 158 & 173 & 71 & 96 & 147 & 147 & 359 & 326 & 208 \\
\hline \multirow[t]{2}{*}{5} & Silent MRA & 495 & 321 & 740 & 489 & 719 & 1023 & 376 & 554 & 439 & 1029 & 1286 & 1288 & 789 \\
\hline & Flow rate & 69 & 42 & 281 & 72 & 166 & 232 & 71 & 108 & 39 & 232 & 375 & 379 & 147 \\
\hline \multirow[t]{2}{*}{6} & Silent MRA & 705 & 578 & 884 & - & 1116 & 1045 & 689 & 674 & 784 & 752 & 1275 & 1180 & 1030 \\
\hline & Flow rate & 85 & 40 & 144 & - & 139 & 131 & 85 & 83 & 98 & 97 & 314 & 233 & 123 \\
\hline \multirow[t]{2}{*}{7} & Silent MRA & 586 & 472 & 1113 & - & 862 & 1014 & 586 & 613 & 813 & 1128 & 1364 & 1195 & 817 \\
\hline & Flow rate & 93 & 62 & 196 & - & 136 & 165 & 67 & 87 & 73 & 150 & 280 & 188 & 129 \\
\hline \multirow[t]{2}{*}{8} & Silent MRA & 554 & 667 & 453 & 675 & 950 & 842 & 1326 & 1087 & 824 & 767 & 1173 & 1114 & 90 \\
\hline & Flow rate & 70 & 85 & 86 & 112 & 182 & 189 & 95 & 73 & 74 & 102 & 312 & 285 & 121 \\
\hline \multirow[t]{2}{*}{9} & Silent MRA & 370 & 552 & 591 & 738 & 1081 & 1095 & 483 & 545 & 730 & 487 & 1152 & 1136 & 953 \\
\hline & Flow rate & 53 & 99 & 122 & 139 & 213 & 188 & 72 & 90 & 153 & 97 & 411 & 319 & 146 \\
\hline \multirow[t]{2}{*}{10} & Silent MRA & 523 & 444 & 334 & 991 & 1006 & 1040 & 632 & 648 & 1121 & 632 & 1016 & 1245 & 1007 \\
\hline & Flow rate & 68 & 48 & 23 & 157 & 144 & 143 & 94 & 97 & 187 & 73 & 145 & 227 & 152 \\
\hline \multirow[t]{2}{*}{11} & Silent MRA & 457 & 448 & 726 & 532 & 962 & 998 & 498 & 439 & 246 & 774 & 1188 & 1132 & 65 \\
\hline & Flow rate & 76 & 71 & 129 & 61 & 218 & 212 & 88 & 80 & 25 & 102 & 452 & 247 & 9 \\
\hline \multirow[t]{2}{*}{12} & Silent MRA & 663 & 741 & 905 & 652 & 948 & 1059 & 672 & 627 & 971 & 721 & 1005 & 1249 & 1146 \\
\hline & Flow rate & 84 & 100 & 158 & 83 & 155 & 233 & 109 & 83 & 128 & 102 & 159 & 192 & 19 \\
\hline \multirow[t]{2}{*}{13} & Silent MRA & 469 & 585 & 540 & 556 & 885 & 1002 & 468 & 559 & 1089 & 349 & 1285 & 1401 & 991 \\
\hline & Flow rate & 59 & 68 & 76 & 78 & 119 & 141 & 52 & 80 & 158 & 46 & 253 & 379 & 194 \\
\hline \multirow[t]{2}{*}{14} & Silent MRA & 532 & 651 & 1035 & 777 & 917 & 829 & 516 & 617 & 702 & 568 & 1261 & 1164 & 671 \\
\hline & Flow rate & 70 & 100 & 195 & 114 & 130 & 126 & 68 & 92 & 92 & 56 & 479 & 259 & 121 \\
\hline \multirow[t]{2}{*}{15} & Silent MRA & 529 & 394 & 669 & 563 & 879 & 865 & 491 & 489 & 828 & 514 & 1202 & 1296 & 875 \\
\hline & Flow rate & 82 & 77 & 138 & 92 & 144 & 131 & 82 & 83 & 124 & 90 & 209 & 294 & 130 \\
\hline \multirow[t]{2}{*}{16} & Silent MRA & 673 & 648 & 672 & 774 & 1099 & 1238 & 700 & 702 & 1064 & 1024 & 1251 & 1353 & 1124 \\
\hline & Flow rate & 81 & 64 & 108 & 110 & 215 & 228 & 102 & 106 & 126 & 125 & 174 & 231 & 159 \\
\hline \multirow[t]{2}{*}{17} & Silent MRA & 624 & 613 & 617 & 506 & 938 & 1036 & 538 & 559 & 506 & 655 & 1202 & 1162 & 707 \\
\hline & Flow rate & 79 & 76 & 112 & 59 & 152 & 180 & 88 & 98 & 74 & 111 & 276 & 245 & 10 \\
\hline
\end{tabular}

The RVA data from participant 01 and the LACA data from participants 06 and 07 were excluded from this study due to excessive motion artifacts Int. Intensity; The unit of flow rate: $\mathrm{ml} /$ minutes

phase dispersion artifacts from turbulent flow can result in diminished signal intensity in a 3D TOF-MRA [4-6]. With the use of zero echo time technique, Silent MRA reduces slow flow related signal dropout, rendering it more reliable than 3D TOF-MRA in evaluating slow flow status such as carotid stenosis. Acute phase of thrombus is reported to show high signal intensity in TOF-MRA especially in the cavernous segment of internal carotid arteries [18, 26, 27]. Silent MRA integrates continuous arterial spin labeling with the generation of angiographic images by subtracting labeled and nonlabeled blood flow images. Presumably the signal change attributable to acute thrombus formation can be subtracted, and static tissue such as thrombus cannot be detected by Silent MRA [18]. Oishi et al. [26] reported high rate of false-positive signals of intra-aneurysmal thrombosis on TOF-MRA and concluded that Silent MRA showed better depiction of intra-aneurysmal embolization status compared 


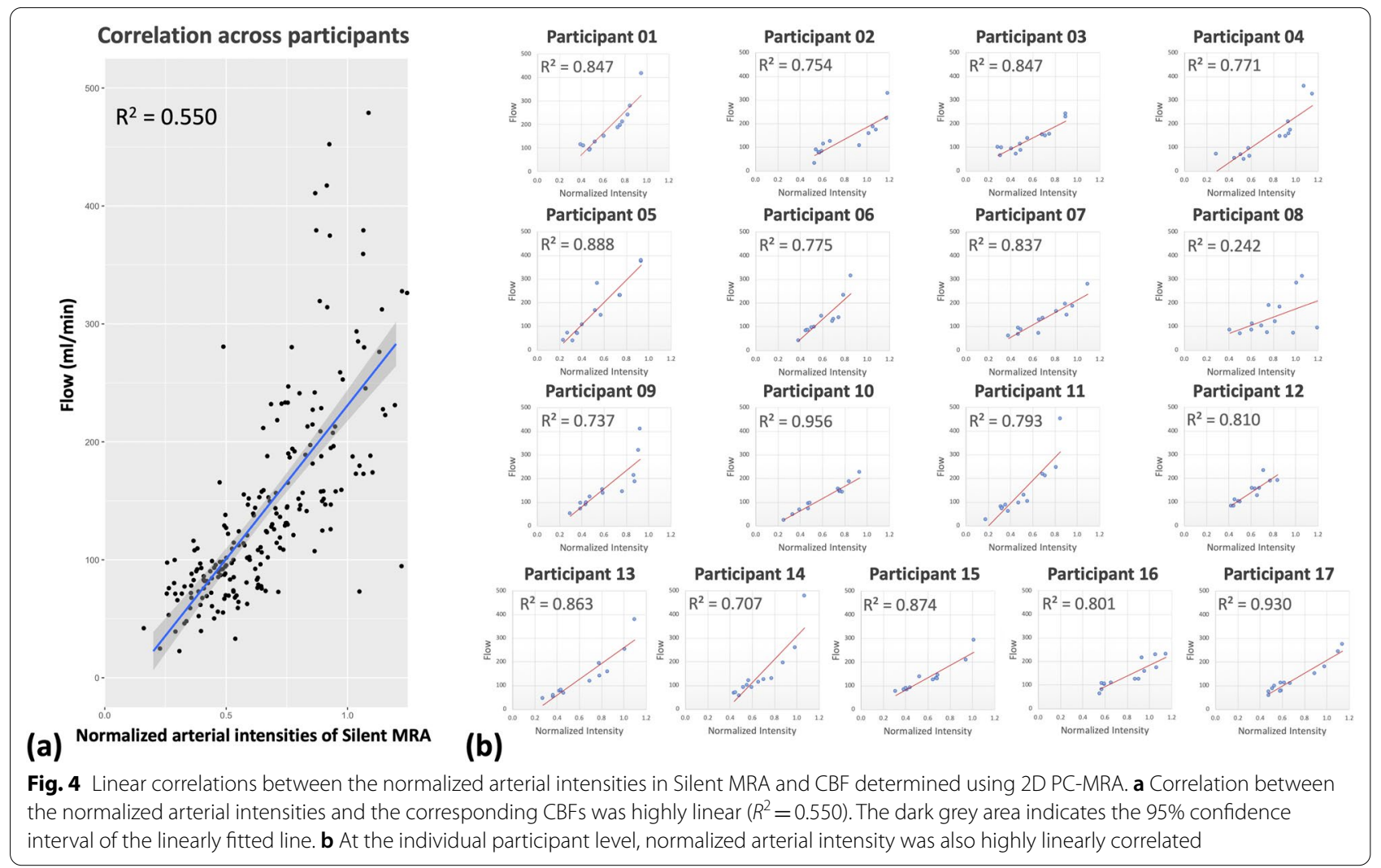

with TOF-MRA. An intracranial stenosis and occlusion attributable to a thrombus could be differentiated using this method. Therefore, Silent MRA can be considered a screening tool for detecting intracranial steno-occlusive disease. Another concerning issue is that the diagnostic role of Silent MRA in chronic small vessel disease has not been well established. Chronic small vessel disease and lacunar infarcts usually involve penetrating arteries and arterioles of Willis' circle. Previous studies showed that Silent MRA was superior to conventional TOF-MRA for visualizing Moyamoya vessels, which referred to the dilated perforating arteries $[28,29]$. Therefore, the potential of Silent MRA in depicting penetrating arteries is worth to be studied.

Cerebral blood flow plays a crucial role in ischemic cerebrovascular disease, and asymmetry in CBF correlates with disease severity and clinical outcome [30]. As this study shows, the signal intensity generated by Silent MRA and an independent CBF measurement are highly positively correlated. The signal intensity seen on the Silent MRA can indirectly imply intraluminal CBF. We presume that a greater intraluminal vessel signal intensity reflects better blood flow in recanalized arteries; therefore, Silent MRA can be applied in acute ischemic stroke to observe arteries following thrombectomy or to determine outcomes following thrombolysis. Recent studies report promising results using Silent MRA as follow-up imaging when assessing coiled or stent-assisted coiled intracranial aneurysms [18-21]. Similarly, stent flow visualization can suggest increased intraluminal CBF despite patency.

Silent MRA has two primary clinical disadvantages. There is marked background suppression with loss of anatomical landmarks, particularly with normal variants of intracranial arteries. However, background suppression shows better blood vessel contrast. Baseline image studies such as computed tomography angiograms or digital subtraction angiographies are performed first and can be used as references, rendering Silent MRA useful as follow-up imaging for treated intracranial arteries. Secondly, compared to 3D TOF-MRA, the longer acquisition time could increase the possibility of motion artifacts.

\section{Limitation}

This study has several limitations. First, it is a singlecenter cohort study with small number participants and fixed scanning parameters; however, it provides promising preliminary results not yet found in the literature. Second, it was limited to young healthy participants. Initially, this was part of the study design to avoid potential bias associated with disease states. Further prospective study with larger population is needed to validate the diagnostic value of Silent MRA in estimating cerebral 
blood flow in older participants with cardiovascular risk or intracranial stenosis. Third, Silent MRA is independent of flow direction. Therefore, cerebral hemodynamic changes with arterial steal and flow reversal, as with subclavian steal syndrome or reversed Robin Hood syndrome, might not be detected. This could be solved by inducing advanced arterial spin labeling such as vesselselective labeling [31, 32]. Fourth, it is difficult to select a common standard from images by Silent MRA for normalization due to the absence of background tissues. The standard used for normalization in this study is the ROI intensity of bilateral ICAs. However, it also contains the flow information within the selected ROIs. This results in the vary correlation between the intensity of Silent MRA and the flow by 2D PC-MRA among participants, which responses to the lower group-leveled result $\left(R^{2}=0.550\right)$ while compared to individual results (most $R^{2}>0.7$ ). It may be solved by a better standard, such as the nonspin-labeling images reconstructed from the individual raw k-space, used for normalization. Fifth, variation of the intracranial artery is not unusual. For example, some degree of asymmetry between the two A1 segments was noted in up to $80 \%$ of cases [33], and the frequency of hypoplastic vertebral artery has been reported in up to $26.5 \%$ of normal healthy population [34]. The distribution of the signal intensity and vessel contrast generated by Silent MRA may result in alterations. For the middle cerebral artery region, we only measured M1 segments bilaterally, and further labeling of the M4 segments should be evaluated in future study. Lastly, Silent MRA is currently not feasible for single artery measurement and does not provide assessment of its CBF quantification.

In conclusion, the normalized arterial intensity in Silent MRA and the corresponding CBF generated by 2D PC-MRA are positively correlated, and the former can be used to predict the range of the latter. By providing a better depiction of the arterial structure and additional CBF information compared to 3D TOF-MRA, Silent MRA is a potential tool for screening steno-occlusive disease and providing supporting imaging information.

\section{Abbreviations}

ASL: Arterial spin labeling; CBF: Cerebral blood flow; MRA: Magnetic resonance angiography; PC: Phase-contrast; SNR: Signal-to-noise ratio; TE: Echo time; TI: Inversion time; TOF: Time-of-flight; TR: Repetition time.

\section{Supplementary Information}

The online version contains supplementary material available at https://doi. org/10.1186/s13244-021-01132-0.

Additional file 1. Figure S1. Maximum intensity projection (MIP) images from TOF-MRA and Silent MRA. Compared with TOF-MRA, Silent MRA provides more uniform signal intensity of bilateral ICAs with less signal loss (yellow arrowheads).

\section{Authors' contributions}

Z-A.H., C-W.L., J-H.C., and W.P.C. made substantial contributions to the study concept and design; Z-A.H., C-W.L., and C-Y.C. acquired and analyzed the data; Z-A.H. provided statistical analyses and interpretation; C-W.L. and C-Y.L. provided technical support; Z-A.H. composed the first draft. All authors provided critical revisions related to important intellectual content, and all approved the final version for publication and agree to be accountable for all aspects of the work and will appropriately investigate and resolve questions related to the accuracy and integrity of any part of the work. All authors read and approved the final manuscript.

\section{Funding}

The authors gratefully acknowledge the receipt of partial financial support from the Inter-agency Research Fund (Grant No. 107-wf-swf-09) and Seed Fund (Grant No. 109-wf-eva-21) Wan Fang Hospital, Taipei Medical University.

\section{Availability of data and materials}

The datasets generated and analyzed during the current study are not publicly available due to the policy of the Research Ethics Committee of Taipei Medical University_-Joint Institutional Review Board but are available from the corresponding author on reasonable request.

\section{Declarations}

Ethics approval and consent to participate

This study was approved by the Research Ethics Committee of Taipei Medical University—Joint Institutional Review Board (N201803017), and informed consent was obtained from all participants.

\section{Consent for publication}

Not applicable.

\section{Competing interests}

Two co-authors, C-W. Li, and C-Y. Lin are employees of GE Healthcare, Taiwan, and they provided sequence information and technical support for the silentMRA software. C-W. Li contributed to the data acquisition and to the Figures illustration based on the results analyzed by Z-A. Hwang. No funding was received from GE Healthcare for publication activities.

\section{Author details}

'Department of Radiology, Wan Fang Hospital, Taipei Medical University, 111 Hsing Long Road, Section 3, Taipei 116, Taiwan. ${ }^{2}$ Department of Radiology, School of Medicine, College of Medicine, Taipei Medical University, Taipei, Taiwan. ${ }^{3} \mathrm{GE}$ Healthcare, Taipei, Taiwan. ${ }^{4}$ Department of Electrical Engineering, National Taiwan University, Taipei, Taiwan. ${ }^{5}$ Neurobiology and Cognitive Science Center, National Taiwan University, Taipei, Taiwan.

Received: 29 July 2021 Accepted: 18 November 2021

Published online: 11 December 2021

\section{References}

1. Wintermark M, Sesay M, Barbier E et al (2005) Comparative overview of brain perfusion imaging techniques. Stroke 36:e83-99. https://doi.org/ 10.1161/01.STR.0000177884.72657.8b

2. Heiserman JE, Drayer BP, Keller PJ, Fram EK (1992) Intracranial vascular stenosis and occlusion: evaluation with three-dimensional time-offlight MR angiography. Radiology 185:667-673. https://doi.org/10. 1148/radiology.185.3.1438743

3. van Amerongen MJ, Boogaarts HD, de Vries J et al (2014) MRA versus DSA for follow-up of coiled intracranial aneurysms: a meta-analysis. AJNR Am J Neuroradiol 35:1655-1661. https://doi.org/10.3174/ajnr. A3700

4. Evans AJ, Richardson DB, Tien R et al (1993) Poststenotic signal loss in MR angiography: effects of echo time, flow compensation, and fractional echo. AJNR Am J Neuroradiol 14:721-729

5. Oshinski JN, Ku DN, Pettigrew RI (1995) Turbulent fluctuation velocity: the most significant determinant of signal loss in stenotic vessels. Magn Reson Med 33:193-199. https://doi.org/10.1002/mrm.1910330208 
6. Anderson CM, Saloner D, Tsuruda JS et al (1990) Artifacts in maximumintensity-projection display of MR angiograms. AJR Am J Roentgenol 154:623-629. https://doi.org/10.2214/ajr.154.3.2106232

7. Amin-Hanjani S, Du X, Pandey DK et al (2015) Effect of age and vascular anatomy on blood flow in major cerebral vessels. J Cereb Blood Flow Metab 35:312-318. https://doi.org/10.1038/jcbfm.2014.203

8. Enzmann DR, Ross MR, Marks MP, Pelc NJ (1994) Blood flow in major cerebral arteries measured by phase-contrast cine MR. AJNR Am J Neuroradiol 15:123-129

9. Zarrinkoob L, Ambarki K, Wåhlin A et al (2015) Blood flow distribution in cerebral arteries. J Cereb Blood Flow Metab 35:648-654. https://doi.org/ 10.1038/jcbfm.2014.241

10. Oelerich M, Lentschig MG, Zunker P et al (1998) Intracranial vascular stenosis and occlusion: comparison of 3D time-of-flight and 3D phasecontrast MR angiography. Neuroradiology 40:567-573. https://doi.org/10. 1007/s002340050645

11. Wu H, BlockWF, Turski PA et al (2014) Noncontrast dynamic 3D intracranial MR angiography using pseudo-continuous arterial spin labeling (PCASL) and accelerated 3D radial acquisition. J Magn Reson Imaging 39:1320-1326. https://doi.org/10.1002/jmri.24279

12. Barger AV, Block WF, Toropov Y et al (2002) Time-resolved contrastenhanced imaging with isotropic resolution and broad coverage using an undersampled 3D projection trajectory. Magn Reson Med 48:297-305. https://doi.org/10.1002/mrm.10212

13. Madio DP, Lowe IJ (1995) Ultra-fast imaging using low flip angles and FIDs. Magn Reson Med 34:525-529. https://doi.org/10.1002/mrm.19103 40407

14. Alsop DC, Detre JA (1998) Multisection cerebral blood flow MR imaging with continuous arterial spin labeling. Radiology 208:410-416. https:// doi.org/10.1148/radiology.208.2.9680569

15. Detre JA, Leigh JS, Williams DS, Koretsky AP (1992) Perfusion imaging. Magn Reson Med 23:37-45. https://doi.org/10.1002/mrm.1910230106

16. Holdsworth SJ, Macpherson SJ, Yeom KW et al (2018) Clinical evaluation of silent T1-weighted MRI and silent MR angiography of the brain. AJR Am J Roentgenol 210:404-411. https://doi.org/10.2214/AJR.17.18247

17. Mizuki K, Masui T, International MKPOJAM (2014) Evaluation of patients of the cerebral vasculature: comparison with Silent MRA and 3D TOF MRA. International Society for Magnetic Resonance in Medicine websitedev. ismrm.org/2014/3882.html

18. Irie R, Suzuki M, Yamamoto M et al (2015) Assessing blood flow in an intracranial stent: a feasibility study of MR angiography using a silent scan after stent-assisted coil embolization for anterior circulation aneurysms. AJNR Am J Neuroradiol 36:967-970. https://doi.org/10.3174/ajnr.A4199

19. Shang S, Ye J, Luo X et al (2017) Follow-up assessment of coiled intracranial aneurysms using zTE MRA as compared with TOF MRA: a preliminary image quality study. Eur Radiol 27:4271-4280. https://doi.org/10.1007/ s00330-017-4794-z

20. Takano N, Suzuki M, Irie R et al (2017) Non-contrast-enhanced silent scan MR angiography of intracranial anterior circulation aneurysms treated with a low-profile visualized intraluminal support device. AJNR Am J Neuroradiol 38:1610-1616. https://doi.org/10.3174/ajnr.A5223

21. Ryu KH, Baek HJ, Moon Jl et al (2020) Usefulness of noncontrastenhanced silent magnetic resonance angiography (MRA) for treated intracranial aneurysm follow-up in comparison with time-of-flight MRA. Neurosurgery 87:220-228. https://doi.org/10.1093/neuros/nyz421

22. Arai N, Akiyama T, Fujiwara K et al (2020) Silent MRA: arterial spin labeling magnetic resonant angiography with ultra-short time echo assessing cerebral arteriovenous malformation. Neuroradiology 62:455-461. https:// doi.org/10.1007/s00234-019-02345-3

23. Tomura N, Saginoya T, Kokubun M et al (2019) Comparison of timeof-flight-magnetic resonance angiography from silent scan magnetic resonance angiography in depiction of arteriovenous malformation of the brain. J Comput Assist Tomogr 43:943-947. https://doi.org/10.1097/ RCT.0000000000000935

24. Fujiwara Y, Muranaka Y (2017) Improvement in visualization of carotid artery uniformity using silent magnetic resonance angiography. Radiol Phys Technol 10:113-120. https://doi.org/10.1007/s12194-016-0375-0

25. Sadikin C, Teng MM-H, Chen T-Y et al (2007) The current role of 1.5T noncontrast 3D time-of-flight magnetic resonance angiography to detect intracranial steno-occlusive disease. J Formos Med Assoc 106:691-699. https://doi.org/10.1016/S0929-6646(08)60030-3
26. Oishi H, Fujii T, Suzuki M et al (2019) Usefulness of silent MR angiography for intracranial aneurysms treated with a flow-diverter device. AJNR Am J Neuroradiol 40:808-814. https://doi.org/10.3174/ajnr.A6047

27. Renard D, Le Bars E, Arquizan C et al (2017) Time-of-flight MR angiography in cerebral venous sinus thrombosis. Acta Neurol Belg 117:837-840. https://doi.org/10.1007/s13760-017-0835-2

28. Fujimura M, Bang OY, Kim JS (2016) Moyamoya disease. Front Neurol Neurosci 40:204-220. https://doi.org/10.1159/000448314

29. Tomura N, Kokubun M, Horiuchi K, Watanabe Z (2019) Comparison of TOF-MRA and silent scan-MRA in depicting cerebral arteries in patients with Moyamoya disease. Acta Radiol 60:1321-1328. https://doi.org/10. $1177 / 0284185118824782$

30. Chalela JA, Alsop DC, Gonzalez-Atavales JB et al (2000) Magnetic resonance perfusion imaging in acute ischemic stroke using continuous arterial spin labeling. Stroke 31:680-687. https://doi.org/10.1161/01.str. 31.3.680

31. Hernandez-Garcia L, Lahiri A, Schollenberger J (2019) Recent progress in ASL. Neuroimage 187:3-16. https://doi.org/10.1016/j.neuroimage.2017. 12.095

32. Wong EC (2007) Vessel-encoded arterial spin-labeling using pseudocontinuous tagging. Magn Reson Med 58:1086-1091. https://doi.org/10. 1002/mrm.21293

33. Mäurer J, Mäurer E, Perneczky A (1991) Surgically verified variations in the A1 segment of the anterior cerebral artery. Report of two cases. J Neurosurg 75:950-953. https://doi.org/10.3171/jns.1991.75.6.0950

34. Park J-H, Kim J-M, Roh J-K (2007) Hypoplastic vertebral artery: frequency and associations with ischaemic stroke territory. J Neurol Neurosurg Psychiatry 78:954-958. https://doi.org/10.1136/jnnp.2006.105767

\section{Publisher's Note}

Springer Nature remains neutral with regard to jurisdictional claims in published maps and institutional affiliations.

\section{Submit your manuscript to a SpringerOpen ${ }^{\circ}$ journal and benefit from:}

- Convenient online submission

- Rigorous peer review

- Open access: articles freely available online

- High visibility within the field

Retaining the copyright to your article

Submit your next manuscript at springeropen.com 